\section{A CASCADE ADD/DROP TRANSCEIVER STRUCTURE TO SOLVE THE OPTICAL BEAT INTERFERENCE PROBLEM IN SUBCARRIER-MULTIPLEXED PASSIVE OPTICAL NETWORKS}

\author{
Wen-Piao Lin, ${ }^{1}$ Ming-Seng Kao, ${ }^{2}$ and Sien $\mathrm{Chi}^{1}$ \\ ${ }^{1}$ Institute of Electro-Optical Engineering \\ National Chiao Tung University \\ Hsin Chu 300, Taiwan, R.O.C. \\ ${ }^{2}$ Department of Communication Engineering \\ National Chiao Tung University \\ Hsin Chu 300, Taiwan, R.O.C.
}

\section{Received 15 October 1999}

ABSTRACT: A cascade add/drop transceiver (CAT) structure in a star-ring architecture for subcarrier-multiplexed passive optical networks (SCM-PONs) is demonstrated to overcome the optical beat interference (OBI) problem. The proposed CAT structure is a simple, reliable, and feasible approach for SCM-PONs. Our experimental results reveal that the CAT structure in a ring can completely eliminate the OBI problem, and maintain a good transmission quality. (C) 2000 John Wiley \& Sons, Inc. Microwave Opt Technol Lett 25: 1-4, 2000.

Key words: cascade add / drop transceiver (CAT); optical beat interference (OBI); optical network units (ONUs); star-ring architecture

\section{INTRODUCTION}

Optical beat interference (OBI) is considered a serious impairment in subcarrier-multiplexed passive optical networks (SCM-PONs) [1-4]. It is the mixing of the optical fields from different optical sources that leads to the OBI problem in the receiver. The OBI will degrade the carrier-to-noise ratio (CNR), and limit the number of optical network units (ONUs). Many useful approaches have been proposed to reduce the influence of OBI. For instance, Woodward, Lu, and Gnauck suggested that different mode spacing lasers could effectively reduce OBI [5]. Besides, our previous work showed that a strong sine-wave modulation could reduce OBI to an acceptable level [6, 7]. However, both of the above-mentioned methods could not completely remove $\mathrm{OBI}$ in the SCM-PONs.

Three basic network architectures-star, bus, and ring-are presented in the PONs. It is shown that, when the network is large, a two-level ring network uses the least amount of fiber. Results indicate that a two-level star network requires at least twice as much fiber as a two-level ring network for 450 or more ONUs. However, a two-level ring network has at least twice the average propagation delay of a star for 280 or more ONUs [8]. Accordingly, a properly optimized two-level network having a star on the upper level and rings on the lower level, as shown in Figure 1, could be a good choice in terms of fiber cost and propagation delay.

In this paper, we propose a CAT structure in passive optical star-ring networks to effectively and systematically solve the OBI problem. This work, being a system approach to deal with the problem, eliminates OBI completely in the low-level ring networks by using the cascaded add/drop transceiver in each ONU. The OBI in the overall system therefore can be greatly reduced for passive star-ring networks. Owing to the simple construction, it is surely a costeffective structure. The implementation of fiber to the home (FTTH), being an inevitable trend in the future, can

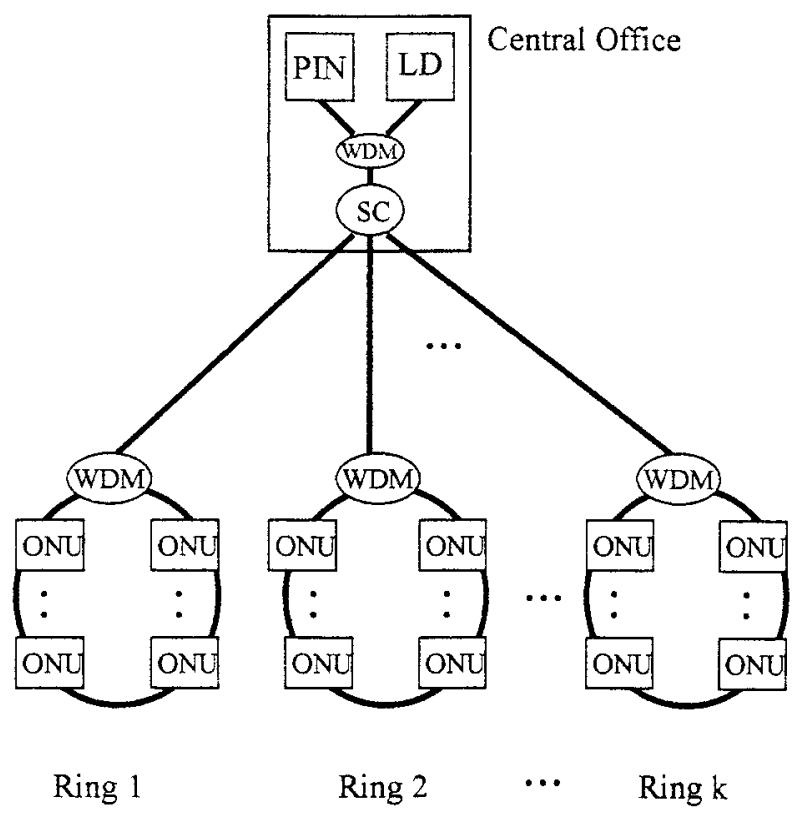

Figure 1 Passive optical star-ring architecture

be realized through the proposed passive optical star-ring structure.

\section{NETWORK ARCHITECTURE}

A bidirectional SCM-based star-ring network using 1.55 $\mu \mathrm{m} / 1.31 \mu \mathrm{m}$ coarse wavelength-division multiplexing (CWDM) is shown in Figure 1. The $1.31 \mu \mathrm{m}$ band is used for upstream transmission, and the $1.55 \mu \mathrm{m}$ band is used for downstream transmission. The host digital terminal (HDT) in the central office contains a $1.55 \mu \mathrm{m}$ laser module and a $p-i-n$ receiver module. This HDT is connected via a singlemode fiber to a star coupler, and each branch is connected to a ring through CWDM. We adopt the CAT structure in each ONU for the ring as shown in Figure 2. In the $\mathrm{ONU}_{i}$, both the downstream optical signal originating from the HDT and the upstream optical signals coming from those ONUs ahead of the $\mathrm{ONU}_{i}\left(\mathrm{ONU}_{1}, \mathrm{ONU}_{2}, \ldots, \mathrm{ONU}_{i-1}\right)$ are sent to a $p-i-n$ module. The downstream signal and the upstream signals occupy the high- and low-frequency bands, respectively. The $p-i-n$ output is connected to an add/drop module, as shown in Figure 3. Using a bandpass filter (BPF), a demultiplexer (DMUX), and an FSK demodulator (DFSK), the downstream signal (digital data or multichannel video signals) can be dropped. On the other hand, the local upstream signal is added before modulating a $1.31 \mu \mathrm{m}$ laser. The laser output is sent to the $\mathrm{ONU}_{i+1}$.

Compared with the clipping tone approach [6], the CAT structure in the star-ring architecture offers four advantages. 1) It is less complicated without a strong clipping tone in each ONU, and it saves many WDM couplers in the optical loop. 2) It completely removes OBI in the ring network because the received optical signal is always from a sole laser source. Therefore, the OBI for the overall system can be reduced to a minimum. 3) This structure has a good reliability, and can be expanded easily due to the cascade ring approach. 4) The upstream signals are regenerated at each ONU, so that a low-cost, low-power laser or LED transmitter can be used at each ONU. 


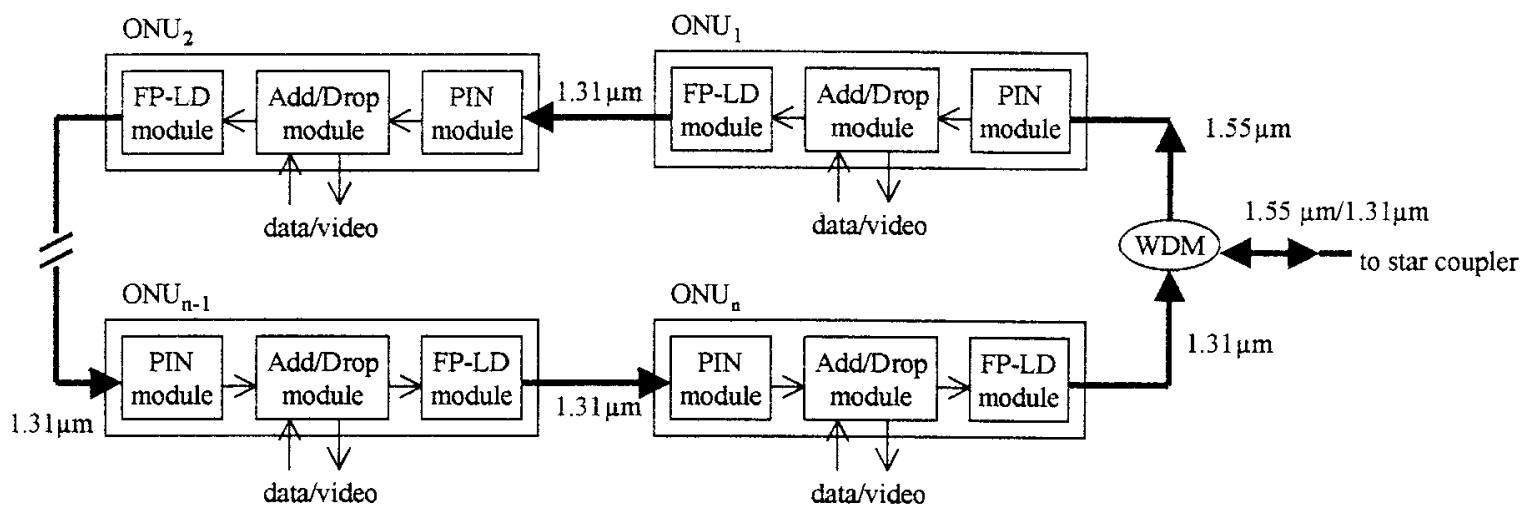

Figure 2 Ring structure

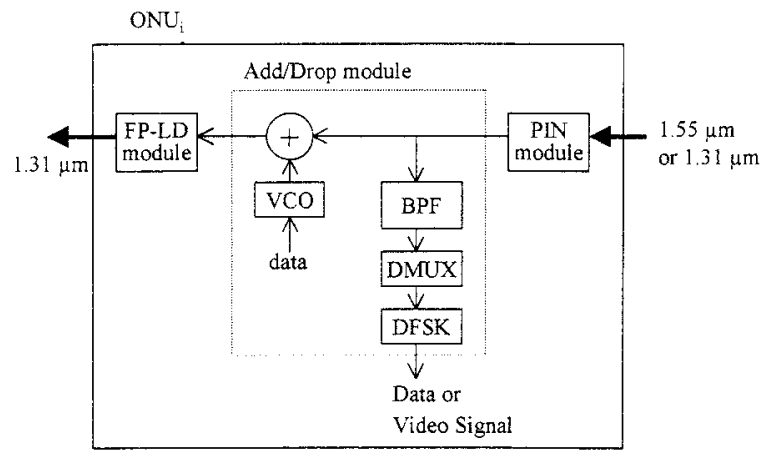

Figure 3 Cascade add/drop transceiver in each ONU

\section{EXPERIMENTAL SETUP AND RESULTS}

Figure 4 shows our experimental setup. In the downstream direction, a $1.55 \mu \mathrm{m}$ uncooled Fabry-Perot laser was directly modulated by a signal consisting of several 3 Mbit/s FSK subcarrier signals and analog FM video channels. The FSK channels are $6 \mathrm{MHz}$ apart, and the spacing for the FM video channels is $38.36 \mathrm{MHz}$. The multiplexer frequency bandwidth covers 450-900 MHz. In the upstream direction, a low-cost $1.31 \mu \mathrm{m}$ uncooled Fabry-Perot laser in the $\mathrm{ONU}_{i}$ was directly modulated with several $3 \mathrm{Mbit} / \mathrm{s}$ FSK subcarrier signals originating from the $(i-1)$ cascade ONUs ahead of the $\mathrm{ONU}_{i}$. The upstream FSK subcarrier channel spacing is $6 \mathrm{MHz}$, and ranges from 80 to $400 \mathrm{MHz}$. The $3 \mathrm{Mbit} / \mathrm{s}$ data signal can be converted into an FSK signal through a VCO module with a modulation index of 0.5. And the output of FSK signals is used to directly modulate the laser, which has an optical modulation index of 0.9 . In this work, the upstream subcarrier channels are 100, 106, 112, and $118 \mathrm{MHz}$, and the $106 \mathrm{MHz}$ channel was monitored during this experiment. In the downstream direction, a $450 \mathrm{MHz}$ subcarrier channel was monitored. The total optical loss through the $2.2 \mathrm{~km}$ singlemode fiber is $0.82 \mathrm{~dB}$ at $1.31 \mu \mathrm{m}$ and $0.43 \mathrm{~dB}$ at $1.55 \mu \mathrm{m}$, respectively. The end-to-end optical loss of the system is $13.52 \mathrm{~dB}$ at $1.31 \mu \mathrm{m}$ and $12.95 \mathrm{~dB}$ at $1.55 \mu \mathrm{m}$.

Figure 5 shows the measured BER of the $450 \mathrm{MHz}$ downstream channel versus the received optical power at the input of the $p-i-n$ module in $\mathrm{ONU}_{1}$. It is monitored under two different cases: 1) only the $450 \mathrm{MHz}$ channel is transmitted, and 2) accompanied by another four SCM channels in

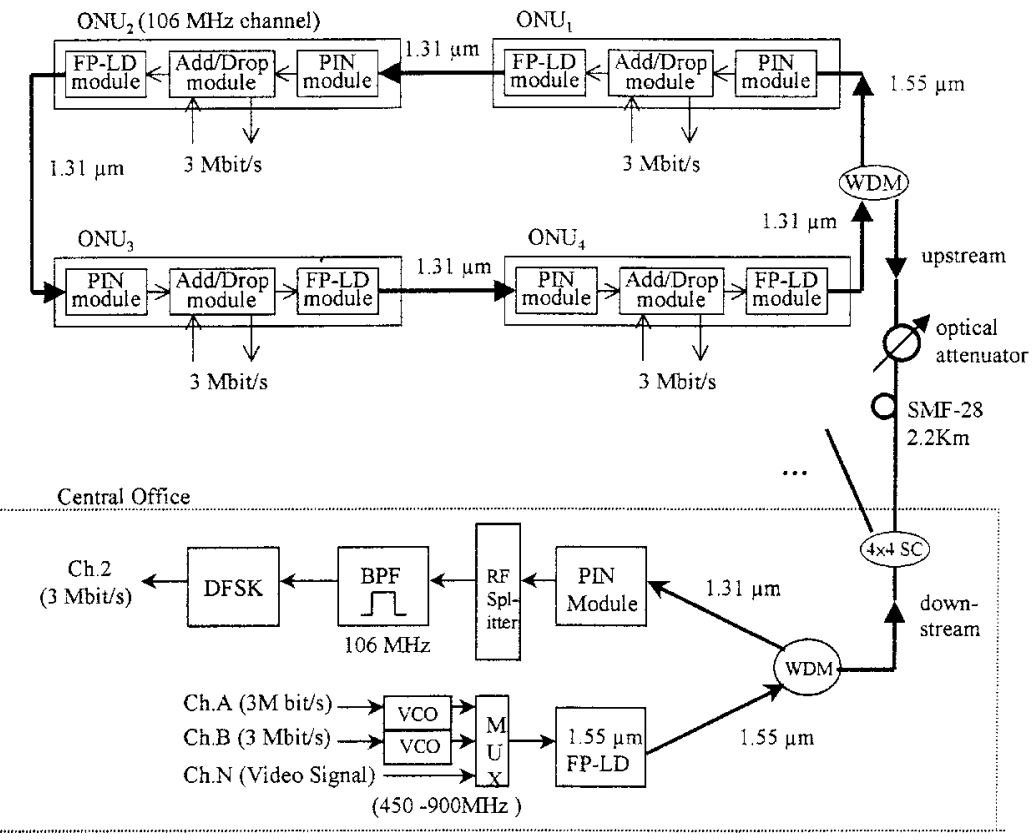

Figure 4 Experimental setup 


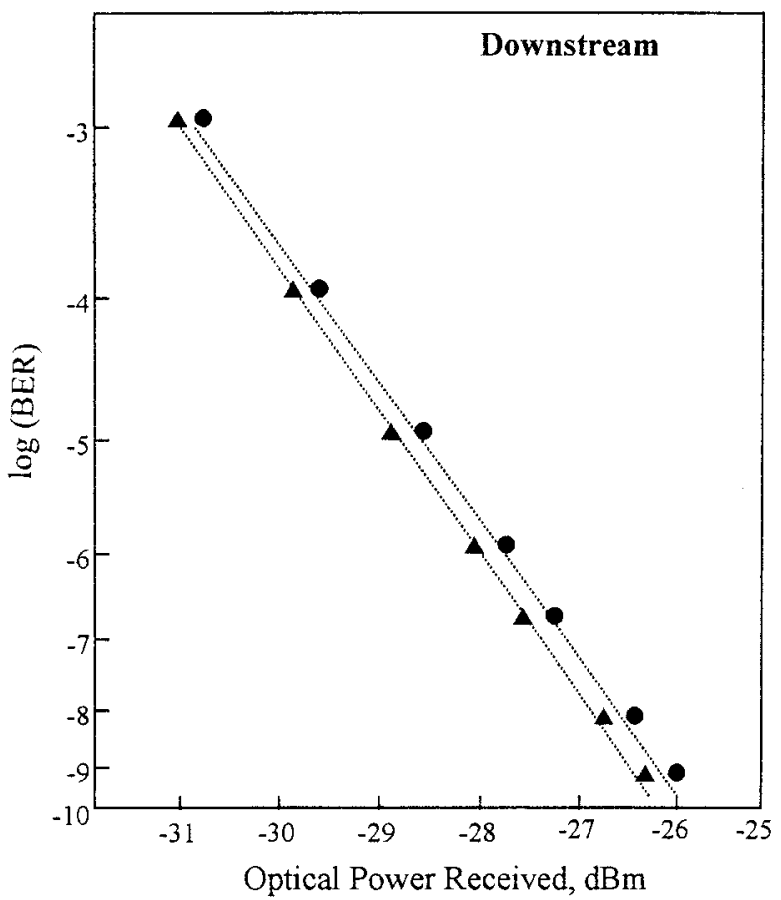

Figure 5 Measured BER curves for downstream transmission on $\mathrm{ONU}_{1}$. \ With one $450 \mathrm{MHz} \mathrm{SCM}$ channel, $\bullet$ with four upstream SCM channels

the upstream direction. For the $1.55 \mu \mathrm{m}$ FP laser in the HDT, the transmitted optical power is $-4 \mathrm{dBm}$, and a BER of $10^{-9}$ is obtained at a received optical power of $-26.4 \mathrm{dBm}$ in case 1). There is a $0.4 \mathrm{~dB}$ power penalty due to the four upstream channels for case 2. Thus, the downstream channel optical power margin is $9.05 \mathrm{~dB}(-4-12.95-0.4+26.4)$. In Figure 6, the $106 \mathrm{MHz}$ upstream channel is monitored under three cases: 1) only the $106 \mathrm{MHz}$ channel is transmitted, 2) three adjacent upstream SCM channels are added, 3) the $450 \mathrm{MHz}$ downstream channel and the three adjacent upstream SCM channels are added. For the $1.31 \mu \mathrm{m}$ FP laser in $\mathrm{ONU}_{4}$, the transmitted optical power is $-4.5 \mathrm{dBm}$, and a BER of $10^{-9}$ is obtained at a receiving optical power of $-29.3 \mathrm{dBm}$ for case 1 ). There is a $0.6 \mathrm{~dB}$ power penalty with three adjacent SCM channels for case 2). Moreover, a downstream channel and three adjacent channel transmission results in a $0.9 \mathrm{~dB}$ power penalty. Thus, the $\mathrm{ONU}_{4}$ optical power margin is $10.38 \mathrm{~dB}(-4.5-13.52-0.9+29.3)$.

\section{DISCUSSION}

Figures 5 and 6 reveal that the optical power penalty is less than $1 \mathrm{~dB}$ for the bidirectional transmission with four upstream channels in a ring and one downstream channel. Cascading many ONUs in a ring and simultaneously having several channels transmitting on the downstream direction can cause interchannel interference (ICI) and accumulated noise problems at the $p-i-n$ module output. Thus, a bottleneck would occur, and both the transmission rate and the maximum number of cascade ONUs in a ring would be limited.

The maximum number of ONUs in the ring is limited by: 1) accumulated noise in the ring, and 2) the modulation bandwidth of the laser. The receiving circuit causes accumulated noise on regenerating the upstream and downstream signals in each ONU. The modulated bandwidth of the laser

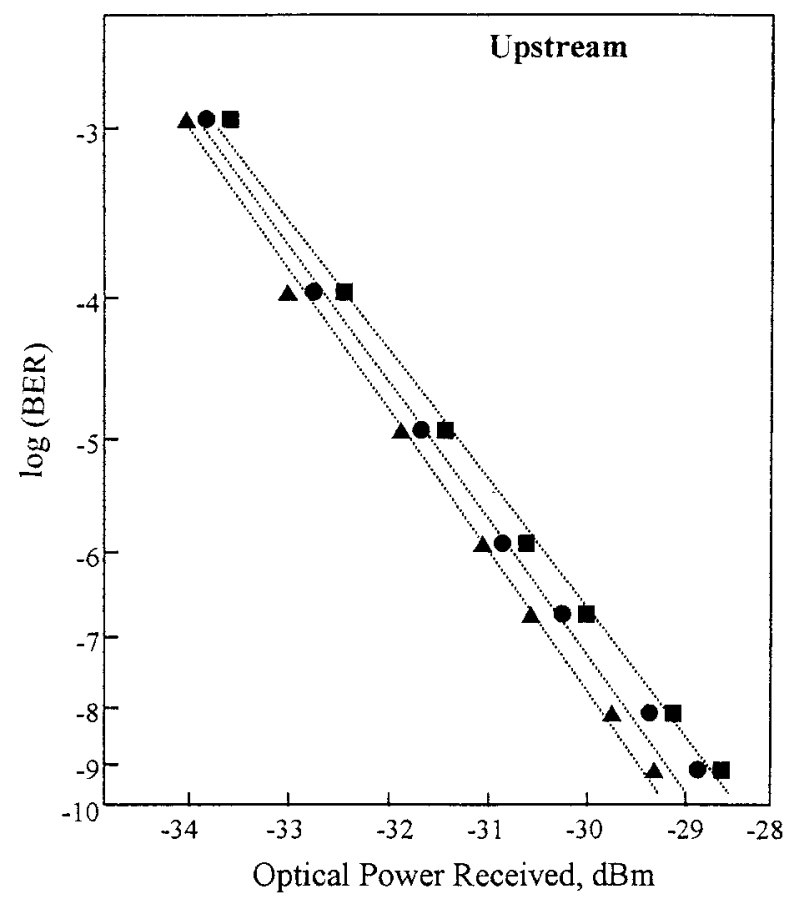

Figure 6 Measured BER curves for upstream transmission on HDT. ^ With one $106 \mathrm{MHz}$ SCM channel, @ with three adjacent SCM channels, - with a downstream SCM channel and three adjacent SCM channels

is obviously a dominant factor for the maximum number of ONUs. In our system, the signals are regenerated in each ONU, and the limitation of the maximum number of ONUs will depend on the laser bandwidth in the last ONU of each ring.

\section{CONCLUSIONS}

We have proposed and demonstrated a star-ring architecture with the CAT approach in dealing with the OBI problem through a system solution for SCM-PONs. Owing to the cascade add/drop transceivers, the optical signals are repeatedly regenerated, and always come from a sole laser source; thereby, the OBI problem can be completely eliminated in each ring. The OBI in the overall system can be greatly reduced. Meanwhile, this architecture is reliable, feasible, and flexible for large networks, being a good candidate for FTTH in the future.

\section{REFERENCES}

1. C. Desem, Optical interference in subcarrier multiplexed system with multiple optical carriers, IEEE J Select Areas Commun 8 (1990), 1290-1295.

2. C. Desem, Measurement of optical interference due to multiple optical carriers in subcarrier multiplexing, IEEE Photon Technol Lett 3 (1991), 387-389.

3. T.H. Wood and N.K. Shankaranarayanan, Operation of a passive optical network with subcarrier multiplexing in the presence of optical beat interference, J Lightwave Technol 11 (1993), $1632-1640$.

4. R.D. Feldman, T.H. Wood, G. Raybon, and R.F. Austin, Effect of optical beat interference on the dynamic range of a subcarrier multiple access passive optical network using Fabry-Perot lasers, J Lightwave Technol 14 (1996), 711-715.

5. S.L. Woodward, X. Lu, and A.H. Gnauck, Bidirectional, subcarrier-multiplexed transmission using $1.3 \mu \mathrm{m}$ Fabry-Perot lasers, IEEE Photon Technol Lett 9 (1997), 1409-1411. 
6. W.P. Lin, Reducing multiple optical carrier interference in broadband passive optical networks, IEEE Photon Technol Lett 9 (1997), 368-370.

7. W.P. Lin, A cost-effective broadband passive optical network achieved by reducing optical beat interference, Microwave Opt Technol Lett 14 (1997), 255-258.

8. K.A. Falcone and O.K. Tonguz, Practical constraints in growth of lightwave networks, IEEE Trans Commun 44 (1996), 348-355.

(c) 2000 John Wiley \& Sons, Inc.

\section{PARALLEL-COUPLED HTS MICROSTRIP ANTENNAS ON ANISOTROPIC SUBSTRATES}

\author{
Adaildo Gomes d'Assunção, ${ }^{1}$ Sandro G. Silva, ${ }^{2}$ and \\ Marcos A. B. Melo \\ ${ }^{1}$ Departamento de Engenharia Elétrica \\ Universidade Federal do Rio Grande do Norte (UFRN) \\ 59072-970 Natal, RN, Brazil \\ ${ }^{2}$ Departamento de Engenharia Elétrica \\ Universidade Federal da Paraíba (UFPB) \\ Campina Grande, PB, Brazil
}

Received 25 October 1999

ABSTRACT: A full-wave analysis of parallel-coupled microstrip antennas with superconducting films on anisotropic substrates is developed in the spectral domain, by using a combination of the moment method, Hertz vector potentials, and the model of two fluids of Gorter and Casimir. The resonant frequencies of coupled superconducting microstrip patches are determined for the even and odd resonating modes, as a function of the temperature and the structural parameters. The radiation pattern for a planar HTS microstrip antenna array is also shown. An excellent agreement was observed between our results and the measured ones, which are available in the literature for single HTS microstrip patch antennas on isotropic substrates. (c) 2000 John Wiley \& Sons, Inc. Microwave Opt Technol Lett 25: 4-8, 2000.

Key words: microstrip antennas; anisotropic substrates; superconducting microstrips; antenna array

\section{INTRODUCTION}

Lately, a growing interest has been observed in the development and use of superconducting materials in microwave and millimeter-wave circuits and systems [1-10].

A special interest has been observed in the use of superconducting materials in microwave integrated circuits [1-8]. This is due to the superconducting material properties, such as small losses (with a reduction of the attenuation and noise level), very small dispersion, even for higher frequencies (dozens of gigahertz), and a reduction in the time of propagation of the signals in the circuits.

The structure of parallel-coupled HTS microstrip antennas is shown in Figure 1. A very thin patch of superconducting material is deposited on a dielectric substrate, which is assumed to be anisotropic and is mounted on a ground plane. The patch superconducting material is a high-temperature superconductor (HTS), with critical temperature $T_{c}$ greater than $77 \mathrm{~K}$

The analysis performed in this paper is a full-wave one, which combines the Galerkin method, Hertz vector potentials, and the two-fluid model of Gorter and Casimir [7-9], in the Fourier domain [10-11].
Curves for the resonant frequency $f_{r}$, are shown as a function of the geometry parameters, including the dielectric and superconducting material properties.

It was observed that the method presented here gives better results than those obtained by using approximate analysis (cavity and modified cavity models). In fact, an excellent agreement was observed between our results and the measured ones reported in the literature for single-HTS microstrip patch antennas [6].

Furthermore, the analysis presented here is quite general, and can be easily extended to investigate the properties of several single and coupled microstrip patch antenna structures, including those with circular, elliptic, ring, and Jerusalem cross patches on multilayered substrates.

\section{THEORY}

Figure 1 shows the structure considered in this work, which is that of two parallel-coupled HTS microstrip patch antennas.

The electric permittivity tensor for dielectric region $j$ $(j=1,2)$, in Figure 1 , is given by

$$
\overline{\bar{\varepsilon}}=\left[\begin{array}{ccc}
\varepsilon_{j 2} & 0 & 0 \\
0 & \varepsilon_{j 1} & 0 \\
0 & 0 & \varepsilon_{j 2}
\end{array}\right] .
$$

To derive the determinantal equation for the complex resonant frequency $f=f_{r}+j f_{i}$, the electric and magnetic Hertz vector potentials are defined as $[10,11]$

$$
\begin{aligned}
& \vec{\pi}_{e}=\pi_{e} \hat{a}_{y j} \\
& \vec{\pi}_{h}=\pi_{h} \hat{a}_{y j}
\end{aligned}
$$

in each of the dielectric regions in Figure 1.

The electric and magnetic fields, in dielectric layer $j$ $(j=1,2)$ for the structure shown in Figure 1, are expressed as a function of the Hertz vector potentials as

$$
\begin{gathered}
\vec{E}=-j \omega \mu_{0} \nabla \times \vec{\pi}_{h j} \\
\vec{H}=j \omega \varepsilon_{0} \nabla \times \vec{\pi}_{e j} .
\end{gathered}
$$

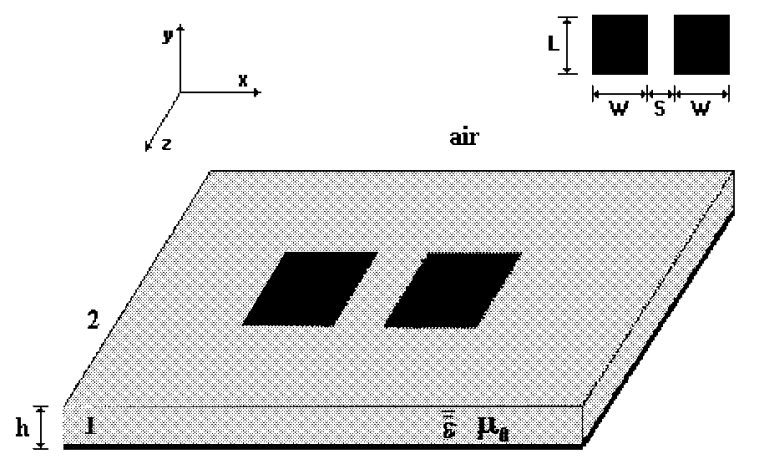

Figure 1 Parallel-coupled HTS microstrip patch resonators on uniaxial anisotropic layer 